\title{
DURAL METASTASIS MIMICKING MENINGIOMA: AN INTERESTING CASE
}

\author{
Hamzaini Abdul Hamid', Kwah Yew Gee ${ }^{1}$, Rohaizak Muhammad², Zurin Adnan Abd Rahman ${ }^{3}$, Srijit Das ${ }^{4}$ \\ National University of Malaysia, Faculty of Medicine, Kuala Lumpur, Malaysia: Department of Radiology ${ }^{1}$, Department \\ of Surgery, Breast and Endocrine Unit ${ }^{2}$, Department of Surgery, Neurosurgery Unit ${ }^{3}$, Department of Anatomy ${ }^{4}$
}

Summary: Dural metastasis is a rare entity in clinical practice. We report a case of dural metastasis secondary to thyroid carcinoma, which on both preoperative CT and MRI and at surgery had the typical appearance of a meningioma. Histopathological findings confirmed metastatic follicular thyroid carcinoma as a primary site. Although rare, dural metastases can mimic a meningioma. Our experience in this case has led us to consider metastasis as a differential diagnosis even when a meningioma is suspected. We believe that reporting of the case of dural metastasis mimicking a meningioma may help clinicians in future.

Key words: Dural; Metastasis; Meningioma; Cancer; Radiology; CT scan; Diagnosis

\section{Introduction}

Meningiomas represent $15 \%$ of all brain tumors. They are the most common extra-axial tumors in the brain. Meningiomas are more common in women than in men (2:1) and have their highest incidence in the sixth and seventh decades (2).

Cerebral metastases are the most frequent brain tumors in adults and they may occasionally present as an isolated meningeal mass, suggesting a meningioma (8). Furthermore, their macroscopic appearance during surgery may even be taken for a meningioma. Because of the prognostic relevance in discriminating both tumors, the definitive diagnosis of a meningioma should be established only after the histopathological report has been analyzed.

\section{Case report}

A 43 year old lady with known medical history of bronchial asthma and hypertension, presented with anterior neck swelling for the past 1 year. She was otherwise well and active. There was no history of dysphagia, hoarseness of voice, loss of weight or loss of appetite.

Physical examination revealed a painless left solitary thyroid nodule and a left hemithyroidectomy was performed. However, the histopathological report showed follicular thyroid carcinoma. She then underwent completion of thyroidectomy. At that time, she also had a sternal mass. A CT thorax was done and showed metastasis to the manubrium and lungs. She was then referred to Nilai Cancer Centre for radiotherapy and has completed 10 cycles. In the thyroid carcinoma treatment, the method of choice also includes the application of radioiodine. This can help also in preoperative diagnosis in the case when isotope scan is made.

She was well until she was admitted again with complaints of persistent headache, blurred vision and weakness of the left leg. A CT scan was performed and it showed a $51 \mathrm{~mm} \times 46 \mathrm{~mm}$ hyperdense well defined mass seen arising from the midline more to the right vertex with surrounding oedema. Following intravenous contrast administration, there was strong enhancement pattern noted. There was no mass effects or midline shift. No intracranial bleed was observed. A diagnosis of cerebral meningioma arising from the falx cerebri was made. She was started on IV Dexamethasone $4 \mathrm{mg}$ TDS and was reduced to $2 \mathrm{mg}$ BD on day 5. The headaches and blurred vision had resolved and the power in her left leg was $4+/ 5$. She was able to ambulate using a walking frame.

Subsequently, the patient was referred to neurosurgical team, National University of Malaysia Medical Centre (UKMMC) for further management. A MRI of the brain showed a well defined lobulated posterior parasagittal lesion. The lesion was isointense to grey matter on $\mathrm{T} 1 \mathrm{~W}$ and $\mathrm{T} 2 \mathrm{~W}$ images with central $\mathrm{T} 1$ hypointensity and $\mathrm{T} 2$ hyperintensity. The lesion was extra-axial in location (as evidence by the CSF sleeve) and compressed the adjacent brain parenchyma. Homogenous enhancement was noted except for the central region with evidence of moderate perilesional oedema. There was evidence of dural tail appearance and enhancement of the falx cerebri. MRI features suggest lesion originating from the meninges at the falx cerebri. The 


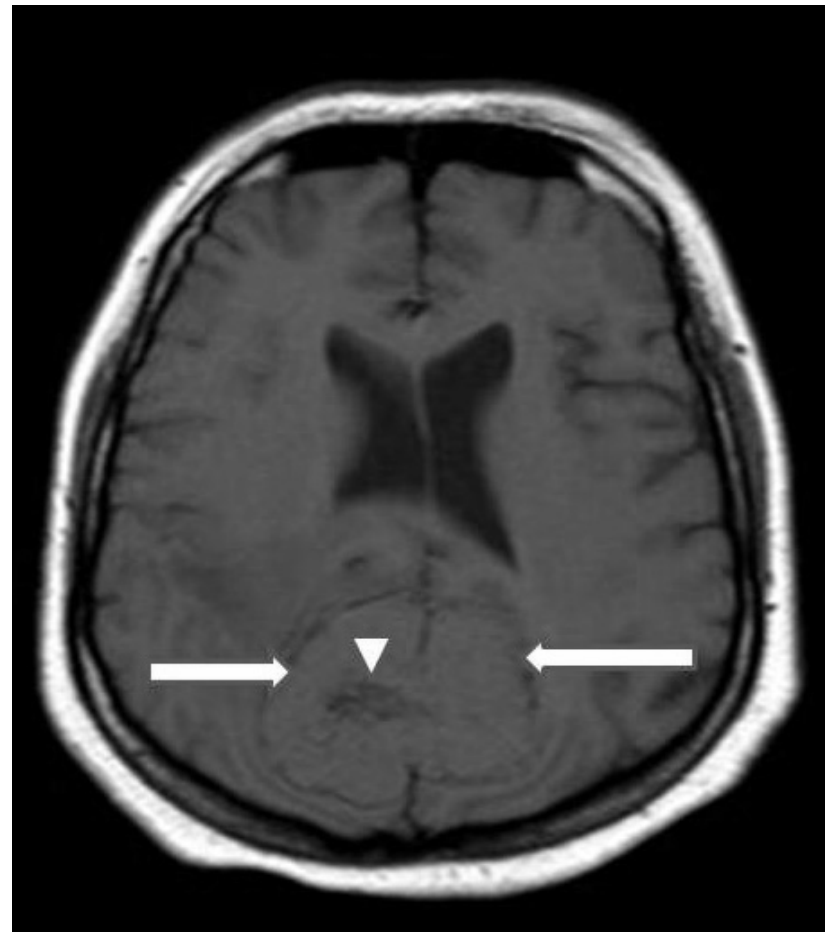

Fig. 1: T1W MRI images show a parasaggital lesion that is isointense to grey matter on $\mathrm{T} 1 \mathrm{~W}$ images (white arrows) with central hypointensity (arrowheads).

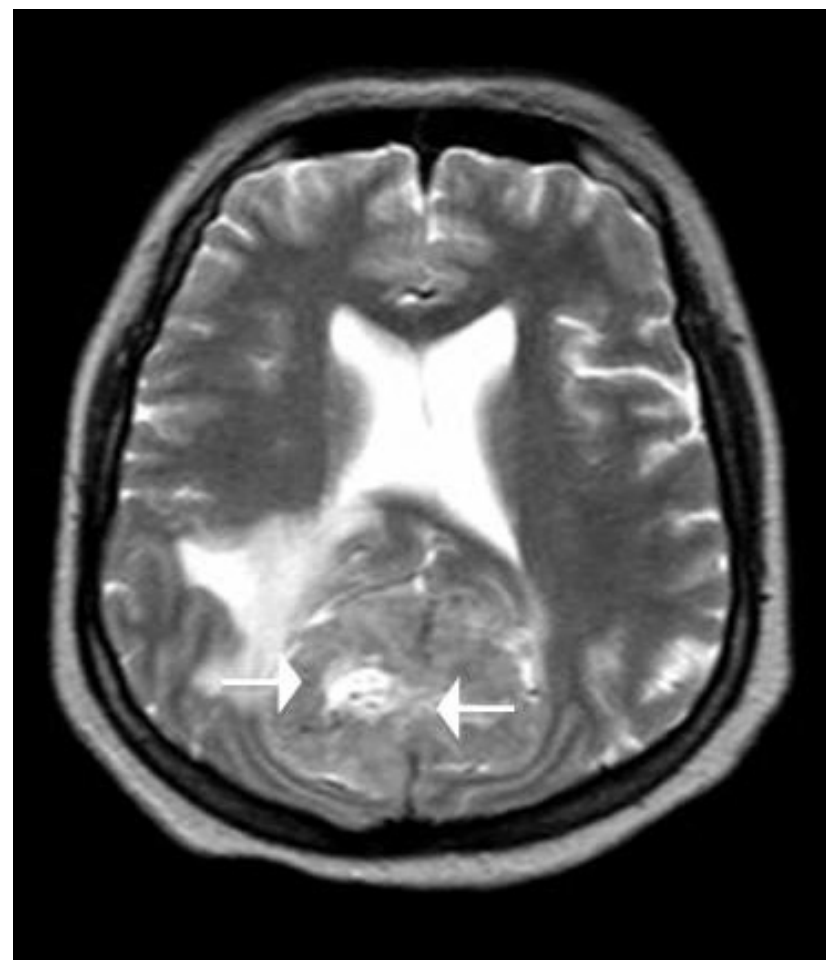

Fig. 2a: T2W images show a well defined lobulated parasaggital lesion (white arrows) at the parietal region, originating from the falx cerebri. diagnosis of meningioma with differential diagnosis of meningeal metastasis was done in view of known primary lesion in the thyroid.

The patient was operated on 6/7/05 with intraoperative findings showed a mixed consistency of yellowish extra-axial tumor originated from the posterior falx. The tumour crossed the midline but spared the superior and inferior saggital sinus. The tumour was totally removed macroscopically. The intra-operative diagnosis was posterior falx meningioma. However, the histopathology revealed metastasis from follicular thyroid carcinoma.

\section{Discussion}

Meningiomas are believed to arise from the arachnoidal (meningothelial) cells, in particular from those forming the arachnoid villi. Grossly, the tumour is firm, gray, and sharply circumscribed, taking the shape of the space in which it grows; thus, some tumors are flat and plaque-like, others round and lobulated. Rarely, they arise from arachnoidal cells within the choroid plexus, forming an intraventricular meningioma.

The usual sites of meningioma are along the falx (parasagittal), dural convexity, sphenoidal ridge, olfactory groove, suprasellar, and posterior fossa. In $10 \%$ of patients the tumors are multiple, and this may be associated with neurofibromatosis type 2 . Histologically, about $85 \%$ are benign.

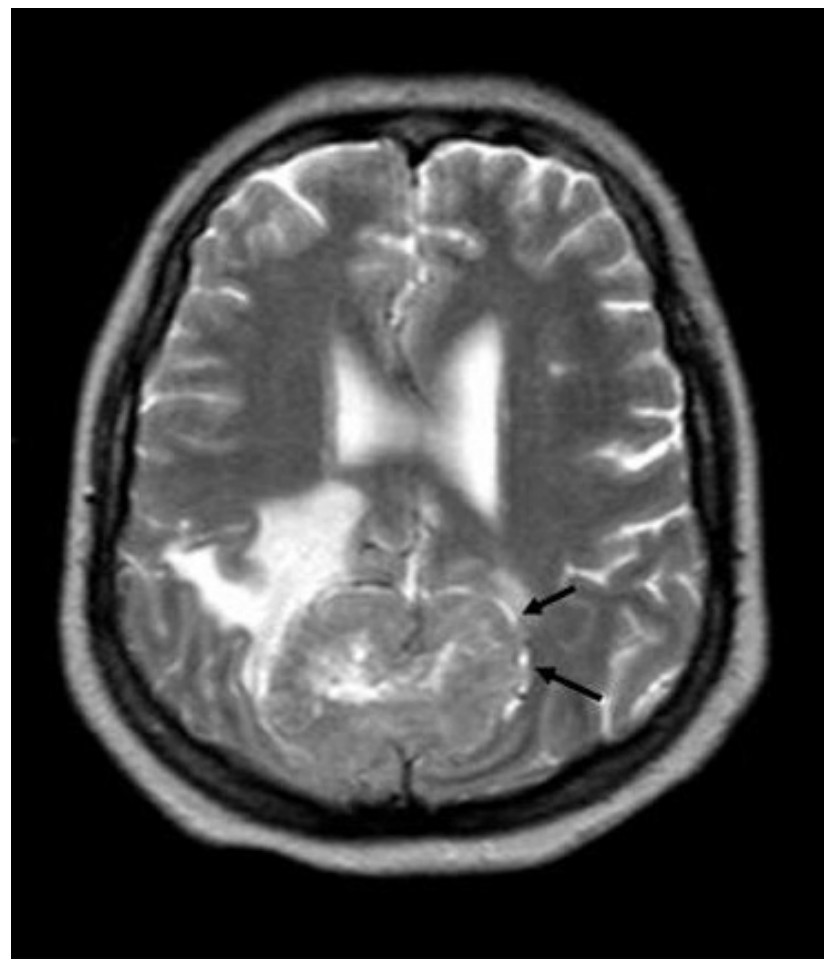

Fig. 2b: The lesion is extraaxial in location as evidence by the hyperintense CSF sleeve (black arrows). 
The typical appearance of meningioma on CT and MRI is well known. Particularly in the elderly, the imaging appearance is sometimes considered diagnostic of these benign tumors without histopathological confirmation (3). Typical appearance of meningiomas in CT findings include sharply demarcated and well circumscribed lesion with a broad base, and isodense-hyperdense to gray matter. CT is effective in showing hyperostosis, bone destruction, and erosion at the site of dural attachment. On MRI, most meningiomas have no signal intensity difference compared with cortical gray matter on non-enhanced T1-weighted images. Meningiomas are iso (50\%) or moderately hyperintense (40\%) on T2-weighted images. On both MRI and $\mathrm{CT}$, meningiomas can exhibit moderate to strong enhancement appearance after the injection of contrast medium.

Direct dural involvement by an underlying intracerebral metastasis is not uncommon (6). However, true dural metastasis are rare and can be mistaken for meningiomas (4). It has been reported in autopsy series that up to $17 \%$ of patients with malignant tumors have intracranial involvement (7) Only $25 \%$ of these metastasis have some degree of meningeal involvement. Isolated meningeal location is seen only in $1 \%$ (7).

Although meningiomas have well known clinical and radiological features, there are reports that point out that metastasis from different neoplasms could simulate them. The most frequently reported primary neoplasms are prostate,

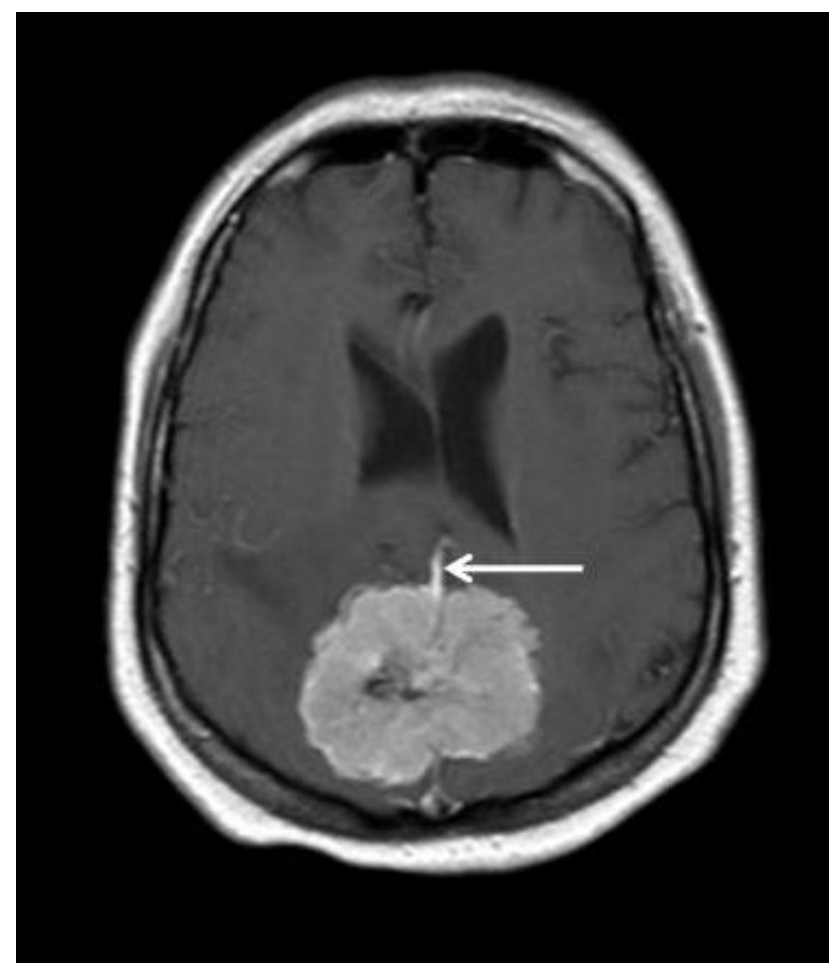

Fig. 4a: Axial image

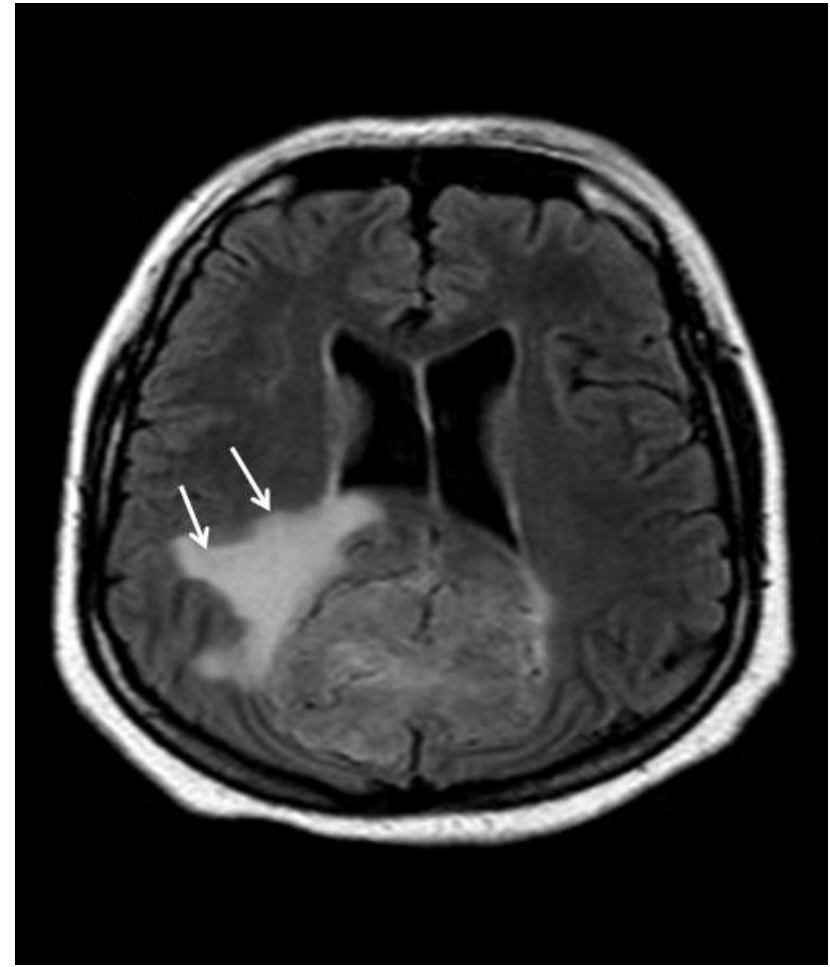

Fig. 3: FLAIR image shows moderate perilesional oedema (white arrows).

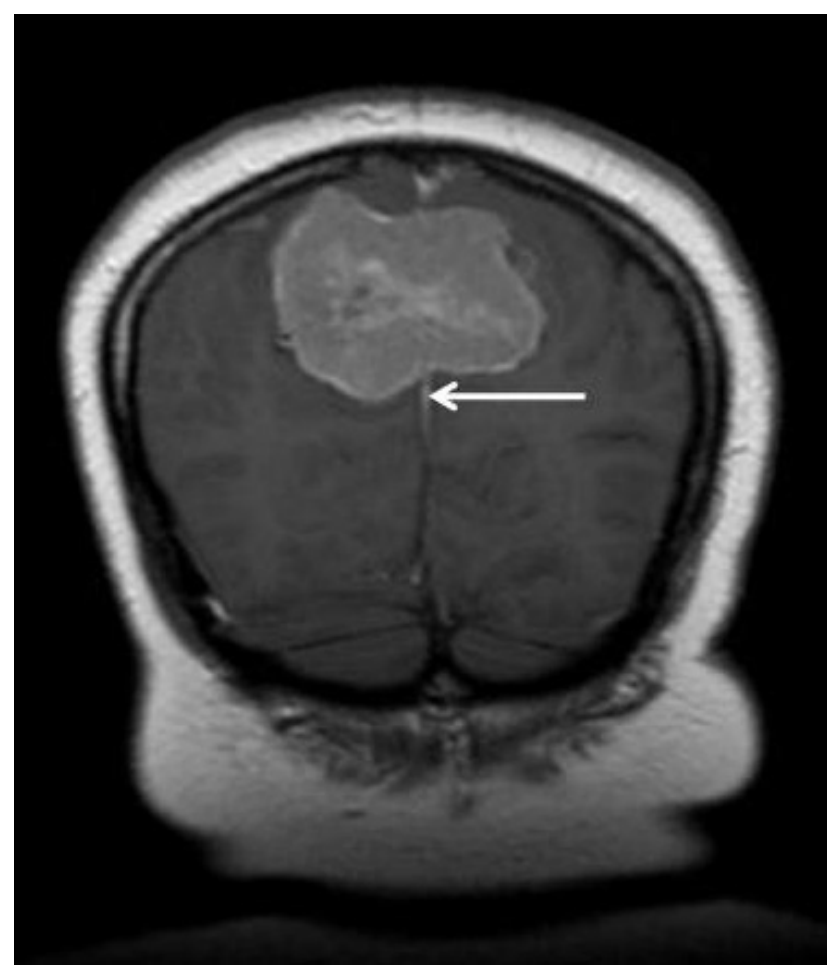

Fig. 4b: Coronal image of post-gadolinium T1W show evidence falx enhancement with dural tail appearance (white arrow). The mass is avidly enhancing. 
kidney, and breast. Dural metastasis from follicular thyroid carcinoma is very rare. Radiological features in this case were those of a meningioma. CT and MRI scan showed a well defined, lobulated, extra-axial, contrast enhancing lesion. The tumor even had the "dural tail" sign. However, "dural tail" sign, which is usually considered to be pathognomonic of meningioma, has also been described with metastatic tumours (5). Based on the radiological findings, diagnosis of a cerebral meningioma with differential diagnosis of meningeal metastasis (from MRI) was made.

The macroscopic appearance of dural metastasis is often indistinguishable from meningioma (8). That also happened in this case, with intra-operative diagnosis of a posterior falx meningioma. Therefore, the definite diagnosis must be histology. The possibility of a metastasis was considered before biopsy in this case because the patient had a history of thyroid primary, although true dural metastases is very rare for this carcinoma.

It may be noted that the experience with cytological examination may not be encouraging in many cases (9). As conclusion, we opine that the dural metastasis can be often mistaken for meningiomas. Although rare, extra-axial duralbased metastasis cannot be reliably differentiated from meningiomas on CT or MRI (1). In patients with known systemic cancer, the radiological finding of an intracranial mass with post contrast enhancement must be considered as a metastasis until proven otherwise and this holds good in the present case, also. Our experience in this case has led us to consider metastasis as a differential diagnosis even when a meningioma is suspected.

\section{References}

1. Bendszus M, Warmuth-Metz M, Burger R, et al. Diagnosing dural metastases: the value of $1 \mathrm{H}$ magnetic resonance spectroscopy. Neuroradiology 2001;43: $285-9$.

2. DeAngelis LM, Gutin PH, Leibel SA, Posner JB. Intracranial tumours: Diagnosis and treatment. London: Martin Dunitz Ltd, 2002:189-205.

3. Laidlaw JD, Kumar A, Chan A. Dural metastases mimicking meningioma. Case report and review of the literature. J Clin Neurosci 2004; 11:780-3.

4. Michael CB, Gokaslan ZL, DeMonte F, et al. Surgical resection of calvarial metastases overlying dural sinuses. Neurosurgery 2001;48:745-54.

5. Quint DJ, McGillicuddy JE. Meningeal metastasis of the cerebellopontine angle demonstrating „dural tail“ sign. Can Assoc Radiol J 1994;45:40-3.

6. Rumana CS, Hess KR, Shi WM, Sawaya R. Metastatic brain tumors with dural extension. J Neurosurg 1998;89:552-8.

7. Simionescu MD. Metastatic tumors of the brain: a follow-up study of 195 patients with neurosurgical considerations. J Neurosurg 1960;17:361-73.

8. Tagle P, Villanueva P, Torrealba G, Huete I. Intracranial metastasis or meningioma? An uncommon clinical diagnostic dilemma. Surg Neurol 2002;58:241-5.

9. Tamiolakis D, Thomaidis V, Tsamis I, et al. Polymorphous low grade adenocarcinoma of the parotid gland. Cytological, histological and immunohistochemical features and review of the literature. Acta Medica (Hradec Kralove) 2004;47:3-6.

Submitted January 2009.

Accepted March 2009.

\section{Corresponding author:}

Dr. Hamzaini Abdul Hamid, Department of Radiology, National University of Malaysia Medical Centre (UKMMC), Bandar Tun Razak, Cheras, 56000. Kuala Lumpur, Malaysia; e-mail: drzanid@yahoo.com 